\title{
A Phase II Study of Weekly Paclitaxel Plus Gemcitabine as a Second-Line Therapy in Patients with Metastatic or Recurrent Small Cell Lung Cancer
}

Tak Yun, MD'
Heung Tae Kim, MD, PhD'1
Ji-Youn Han, MD ${ }^{1}$
Sung Jin Yoon, RN ${ }^{1}$
Hyae Young Kim, MD
Byung-Ho Nam, PhD²
Jin Soo Lee, MD

${ }^{1}$ Center for Lung Cancer, ${ }^{2}$ Cancer Biostatistics Branch, Research Institute and Hospital, National Cancer Center, Goyang, Korea

Correspondence: Heung Tae Kim, MD, PhD Center for Lung Cancer, National Cancer Center, 323 Ilsan-ro, Ilsandong-gu, Goyang 10408, Korea Tel: 82-31-920-1622

Fax: 82-31-920-1520

E-mail: htkim@ncc.re.kr

Received February 16, 2015

Accepted April 14, 2015

Published Online May 26, 2015

*Presented at the poster discussion session of the European Multidisciplinary Conference in Thoracic Oncology, May 1-3, 2009.

\begin{abstract}
Purpose
Paclitaxel $(P)$ and gemcitabine $(G)$ are clinically synergistic in small cell lung cancer (SCLC). We evaluated the efficacy of PG as a salvage treatment for SCLC patients whose disease progressed after a platinum-containing regimen.
\end{abstract}

\section{Materials and Methods}

Eligibility included histologically confirmed SCLC, one dimensionally measurable disease, Eastern Cooperative Oncology Group performance status 0-2, and progressive disease after platinum-based chemotherapy. Treatment consisted of $P\left(80 \mathrm{mg} / \mathrm{m}^{2}\right)$ and $\mathrm{G}\left(1,000 \mathrm{mg} / \mathrm{m}^{2}\right)$ on days 1 and 8 of each cycle of 21 days until disease progression.

\section{Results}

Thirty-three patients seen between December 2005 and February 2009 were selected into this study. Thirty patients (91\%) had received irinotecan-platinum, and three had received etoposide-platinum. Sixteen patients (49\%) had a treatment-free interval of less than 3 months. The overall response rate was $30.3 \%$ (29.4\% in sensitive relapse and $31.3 \%$ in refractory relapse). The median time to progression was 12.0 weeks and median overall survival (OS) 31.0 weeks, with a 1-year OS rate of 30.3\%. Toxicities were moderate and manageable with $18.2 \%$ grade $(\mathrm{G}) 4$ neutropenia, 24.2\% G3 thrombocytopenia, $6.1 \%$ G3 sensory neuropathy, and 3\% G3 asthenia. One patient developed febrile neutropenia.

\section{Conclusion}

Second-line paclitaxel and gemcitabine were well-tolerated and moderately active in SCLC patients previously treated with platinum-based chemotherapy.

Key words

Second-line, Paclitaxel, Gemcitabine, Small cell lung carcinoma

\section{Introduction}

Most patients with small cell lung cancer (SCLC) relapse within a year of initial therapy, and many are candidates for second-line treatment. Although many patients are in excellent physical condition at the time of relapse, few drugs or drug combinations are capable of achieving tumor regression in this setting. Patients with sensitive relapse may respond to a number of agents including topotecan, irinotecan, vinorelbine, paclitaxel, and gemcitabine [1-4]. Topotecan is the best-documented second-line therapy for previouslytreated SCLC, although treatment outcomes have been disappointing. Topotecan produces an $18 \%$ response in patients with sensitive relapse, and in refractory patients, the response is less than $10 \%$ [5,6]. A topotecan/ cisplatin doublet showed a higher response rate in patients with sensitive relapse although median survival times were similar to those obtained with topotecan alone [7]. For some patients with sensitive relapse, re-induction with the same regimen or 
topotecan might be considered, but for patients with refractory relapse, no regimen is a standard option. Thus, patients with good performance status are candidates for clinical trials for further treatment.

As a single agent, paclitaxel produces a response rate of $27 \%$ in SCLC patients with sensitive relapse and a response rate of $20 \%$ in those with refractory relapse [8]. Gemcitabine has a response rate of $16 \%$ in SCLC patients with sensitive relapse and a response rate of $6 \%-13 \%$ in those with resistant relapse $[4,9]$. Because of their single-agent activity, different mechanism of action, non-overlapping toxicities, in vitro synergy, and beneficial pharmacologic interaction, the use of a combination of paclitaxel and gemcitabine is an attractive option [10,11]. Paclitaxel significantly decreases the systemic clearance and volume of distribution of gemcitabine, and significantly increased steady-state concentrations of gemcitabine. Interpatient and intrapatient variability in gemcitabine pharmacokinetics was not observed when gemcitabine was administered in combination with paclitaxel in non-small cell lung cancer (NSCLC) patients [11]. Some phase II trials of first-line gemcitabine plus paclitaxel in NSCLC patients showed encouraging response rates of $41 \%$ $53 \%$ [12-14]. These combination chemotherapies, in which paclitaxel was administered every 3 weeks, were associated with substantial toxicity, and grade 3-4 neutropenia developed in 70\%-80\% of patients. Weekly paclitaxel treatment has an efficacy comparable to that of the every-3-weekly paclitaxel but with less myelosuppression and neurotoxicity.

Based on these studies, we selected a combination of weekly paclitaxel $\left(80 \mathrm{mg} / \mathrm{m}^{2}\right)$ and gemcitabine $(1,000$ $\mathrm{mg} / \mathrm{m}^{2}$ ) and conducted a phase II study to evaluate the efficacy and safety of this combination in patients with SCLC who have relapsed or failed to respond to first-line platinum-based therapy.

\section{Materials and Methods}

\section{Eligibility criteria}

Eligibility in the study required histologically- or cytologically-confirmed SCLC that progressed during or after firstline chemotherapy or chemoradiotherapy. Patients needed to have measurable disease according to the Response Evaluation Criteria in Solid Tumors (RECIST) [15]. Additional criteria were as follows: patients must (1) be at least 18 years old; (2) have an Eastern Cooperative Oncology Group performance status (ECOG PS) of 0 to 2; (3) have not had prior radiotherapy on measurable lesions (but previous surgery and/or chest radiotherapy for the primary lesion was allowed); (4) have had a minimum of 28 days since any prior chemotherapy or radiation before study entry; and (5) have adequate bone marrow, hepatic, and renal functions, defined as a white blood cell count $\geq 3,500 / \mathrm{mm}^{3}$, absolute neutrophil count $(A N C) \geq 1,500 / \mathrm{mm}^{3}$, platelets $\geq 100,000 / \mathrm{mm}^{3}$, alanine aminotransferase or aspartate aminotransferase $\leq 2.5$ times the upper normal limit, serum bilirubin $<1.5$ times the upper normal limit, and serum creatinine $\leq 1.5 \mathrm{mg} / \mathrm{dL}$. Patients were excluded if there was severe comorbidity such as a myocardial infarction within the preceding six months, symptomatic heart disease (including unstable angina, congestive heart failure, or uncontrolled arrhythmia), or serious concomitant infection, including post obstructive pneumonia. The presence of central nervous system (CNS) metastases, however, was not an exclusion criterion, provided that CNS symptoms were absent or sufficiently minor to be wellcontrolled with corticosteroids. Written informed consent approved by the Institutional Review Board of the National Cancer Center was obtained from all patients prior to entering the study. The study followed the Declaration of Helsinki and good clinical practice guidelines. This study was registered with ClinicalTrials.gov (identifier: NCT00453167).

\section{Assessment}

A complete history and physical examination, including documentation of concomitant medications and performance status, standard laboratory studies, and an electrocardiogram, was performed within 14 days prior to study entry. Chest X-ray, computed tomography scans of the chest including the upper abdomen, magnetic resonance imaging of the brain, and a radionuclide bone scan were performed within 4 weeks prior to study entry. Complete blood cell counts and chemistry were performed on days 1 and 8 of each cycle. Objective tumor response was assessed every two cycles according to the RECIST criteria [15]. Toxicity was graded by the National Cancer Institute Common Toxicity Criteria ver. 3.0.

\section{Treatment}

Paclitaxel (Padexol, Seoul, Korea) and gemcitabine (Geroam, Seoul, Korea) were administered to patients. Treatment consisted of $80 \mathrm{mg} / \mathrm{m}^{2}$ paclitaxel and 1,000 mg/ $\mathrm{m}^{2}$ gemcitabine by intravenous infusion on days 1 and 8 every 3 weeks until disease progression. Paclitaxel was given first as a 60-minute intravenous infusion, immediately followed by a 30-minute gemcitabine intravenously. Premedication consisted of dexamethasone (10-20 mg intravenously), pheniramine maleate ( $45.5 \mathrm{mg}$ intravenously), and famotidine (20 mg intravenously) before paclitaxel infusion. Granisetron $(3 \mathrm{mg}$ ) was given intravenously before paclitaxel infusion. 
During the course of chemotherapy, paclitaxel doses were decreased by $15 \mathrm{mg} / \mathrm{m}^{2}$ for grade 4 hematologic toxicities or grade 3 or 4 non-hematologic toxicities. Gemcitabine doses were decreased by $20 \%$ for grade 4 hematologic toxicities or grade 3 or 4 non-hematologic toxicities. The dose reduction was maintained in the subsequent cycles.

\section{Statistical analysis}

The primary objective of this study was to estimate the overall response rate of paclitaxel and gemcitabine as second-line chemotherapy in patients with metastatic or recurrent SCLC. The secondary objectives were to estimate the time to progression (TTP) and overall survival (OS). Simon's minimax two-stage design was used to determine the sample size [16]. The undesirable response rate was $20 \%$ and the desirable expected response rate was $40 \%$. With $80 \%$ power and a $5 \%$ one-sided type I error, 18 patients were needed in the first stage, and 33 patients were needed in total. At the first stage, if there were five or fewer responses out of the initial 18 patients, the study would conclude that the anticipated response rate is less than $20 \%$ and would terminate. Otherwise, accrual would continue to a full sample of 33 assessable patients. At the second stage, at least 11 objective responses among 33 patients were required for this regimen to be regarded as worthy of further investigation. The response rate of the treatment was calculated as the ratio of the number of complete and partial responders to the total number of evaluable patients. A $95 \%$ confidence interval for the response rate was computed based on the binomial distribution function. The toxicity profile was estimated as the ratio of the number of occurrence to the total number of evaluable patients. The TTP and OS were estimated by the Kaplan-Meier method. The OS was defined as the period of time from the first day of treatment to death from any cause. Patients still alive were censored at the last day on which they were known to be alive. The TTP was defined from the first day of treatment to the date that disease progression was assessed. The duration of response for all responders was defined as the period of time from the date of the first response, i.e., either partial response or complete response (whichever occurred first), to the date of disease progression.

\section{Results}

\section{Patient characteristics}

Between December 2005 and February 2009, 33 patients were prospectively enrolled. Patient characteristics are shown in Table 1 . The median age was 65 years (range, 38 to 77 years). Thirty patients (91\%) were men, and 32 patients $(97 \%)$ had an ECOG PS of 0 or 1 . Three patients (9\%) had never smoked. Twenty-three patients $(70 \%)$ had extensive disease at diagnosis. All patients received platinum doublets. Thirty patients $(91 \%)$ received irinotecan-platinum and three patients received etoposide-platinum. As a prior therapy, 23 patients received chemotherapy alone, and 10 patients received chemoradiation. Among the chemotherapy-only group, all 23 patients received irinotecan-platinum chemotherapy. Among the 10 patients treated with chemoradiation, seven patients received irinotecan-platinum, and three patients received etoposide-platinum. Sensitive relapse was defined as a chemotherapy-free interval of 3 months or greater, and refractory relapse was defined as no response to initial therapy or progression within 3 months after initial therapy. Sixteen patients $(49 \%)$ had a refractory relapse.

\section{Treatment exposure}

The median number of cycles administered was three (range, 1 to 12 cycles), and 10 patients completed six cycles of chemotherapy. Over 133 cycles, delayed chemotherapy administration occurred in 44 cycles (33\%), and dose reduction occurred in 22 cycles (16\%). The most common cause for the delay in chemotherapy administration was either holiday (23 cycles) or neutropenia (19 cycles). Twenty-two cycles required dose reduction, mainly because of neutropenia (18 cycles), thrombocytopenia (3 cycles), and pneumonia ( 1 cycle). The average relative dose intensity was $86.4 \%$ for paclitaxel and $85.4 \%$ for gemcitabine.

\section{Efficacy}

No complete response (CR) was observed. Ten patients achieved partial response (PR). The overall response rate was $29.4 \%$ in sensitive relapse and $31.3 \%$ in refractory relapse (Table 2). All patients were tracked until death. The median TTP was 12.0 weeks (95\% confidence interval [CI], 7.57 to 16.44), the median OS was 31.0 weeks (95\% CI, 23.44 to $38.56)$, and the 1-year OS rate was $30.3 \%$ (95\% CI, 14.6 to 46.0$)$ (Fig. 1). No pretreatment characteristic, including sex (male vs. female), ECOG PS (0 vs. 1-2), stage at diagnosis (limited vs. extensive), smoking history (never vs. ever), prior treatment (chemotherapy only, concurrent chemoradiotherapy vs. chemotherapy followed by radiotherapy), best response of any prior treatment (PR vs. non-PR), treatment-free interval ( $<3$ months vs. $3-6$ months vs. $\geq 6$ months), was predictive of tumor response (Table 3 ). Twenty patients received salvage chemotherapy after disease progression on PG chemotherapy. The salvage treatments included cyclophosphamide-adriamycin-vincristine (6 patients), adriamycin- 
Table 1. Patient characteristics

\begin{tabular}{|c|c|}
\hline Characteristic & $\begin{array}{l}\text { No. of patients }(\%) \\
(\mathbf{n}=33)\end{array}$ \\
\hline \multicolumn{2}{|l|}{ Sex } \\
\hline Male & $30(90.9)$ \\
\hline Female & $3(9.1)$ \\
\hline Age, median (range, yr) & $65(38-77)$ \\
\hline \multicolumn{2}{|l|}{ ECOG PS } \\
\hline 0 & $6(18.2)$ \\
\hline 1 & $26(78.8)$ \\
\hline 2 & $1(3.0)$ \\
\hline \multicolumn{2}{|l|}{ Stage (at enrollment) } \\
\hline Recurrence & $25(75.8)$ \\
\hline Metastatic & $8(24.2)$ \\
\hline \multicolumn{2}{|l|}{ Smoking history } \\
\hline Never & $3(9.1)$ \\
\hline Former & $8(24.2)$ \\
\hline Current & $22(66.7)$ \\
\hline \multicolumn{2}{|c|}{ Prior treated anticancer therapy } \\
\hline Chemotherapy only & $23(69.7)$ \\
\hline CCRT & $7(21.2)$ \\
\hline Chemo $\rightarrow$ RT & $3(9.1)$ \\
\hline \multicolumn{2}{|l|}{ Prior chemotherapy } \\
\hline Irinotecan/platinum & $30(90.9)$ \\
\hline Etoposide/platinum & $3(9.1)$ \\
\hline \multicolumn{2}{|c|}{ Best response of prior treatments } \\
\hline $\mathrm{PR}+\mathrm{CR}$ & $27+2(87.8)$ \\
\hline SD & $2(6.1)$ \\
\hline PD & $2(6.1)$ \\
\hline \multicolumn{2}{|c|}{ Chemotherapy-free interval (mo) $\left.{ }^{a}\right)$} \\
\hline$\leq 3$ & $16(48.5)$ \\
\hline$>3$ & $17(51.5)$ \\
\hline
\end{tabular}

CCRT, concurrent chemoradiotherapy; RT, radiotherapy; $\mathrm{PR}$, partial response; $\mathrm{CR}$, complete response; $\mathrm{SD}$, stable disease; $\mathrm{PD}$, progressive disease. ${ }^{\mathrm{a})} \mathrm{Chemotherapy-free}$ interval: from the last date of prior chemotherapy to start date of paclitaxel-gemcitabine.

ifosfamide-vincristine (5 patients), etoposide-cisplatin (3 patients), irinotecan-cisplatin (2 patients), oral etoposide
(2 patients), adriamycin-ifosfamide (1 patient), and pemetrexed (1 patient).

\section{Toxicity}

All patients ( $\mathrm{n}=33$ ) who received at least one cycle of therapy were assessable for toxicity. Toxicity was reported as the maximum toxicity experienced during entire study treatment, rather than just during the first cycle. Hematologic and non-hematologic toxicities observed over the entire course of the study are summarized in Table 4 . Overall toxicities were moderate and manageable. The most common grade (G) 3 or 4 toxicity was myelosuppression. G4 neutropenia was observed in $18.2 \%$ of patients and G3 thrombocytopenia was observed in $24.2 \%$ of patients. Only one patient developed febrile neutropenia. Another patient died of pneumonia during the third cycle, but G1 neutropenia was present (ANC, 1,219/ $\mathrm{L}$ ). G3 sensory neuropathy developed in two patients, and their chemotherapy was stopped. One patient developed G3 neuropathy after the sixth cycle. Another patient developed G3 neuropathy after the first cycle, but recovered completely in 3 months. One patient developed G3 asthenia after the second cycle, but PD was confirmed.

\section{Discussion}

This study was a single-arm phase II study to evaluate the efficacy of paclitaxel plus gemcitabine in patients with SCLC that progressed or failed to respond to platinum-based chemotherapy. Historically, the median OS is only 14 weeks if no active therapy is given other than best supportive care (BSC) after first-line chemotherapy [17]. Second-line chemotherapy increased OS and resulted in better symptom control compared with BSC [17]. The strongest predictor of outcome for patients with relapsed SCLC is the duration of remission. Patients with sensitive disease respond to the same initial therapy in approximately $50 \%$ of cases. The median OS from the start of a second-line therapy is approximately 6 months. In patients with refractory disease,

Table 2. Tumor response

\begin{tabular}{lccc} 
& Sensitive relapse $>\mathbf{3}$ mo $(\mathbf{n}=\mathbf{1 7})$ & Refractory relapse $\leq \mathbf{3}$ mo $(\mathbf{n}=\mathbf{1 6})$ & Total $(\mathbf{n}=\mathbf{3 3})$ \\
PR & $5(29.4)$ & $5(31.3)$ & $10(30.3)$ \\
SD & $5(29.4)$ & $3(18.7)$ & $8(24.2)$ \\
PD & $7(41.2)$ & $8(50.0)$ & $15(45.5)$ \\
\hline
\end{tabular}

Values are presented as number (\%). PR, partial response; SD, stable disease; PD, progressive disease. 


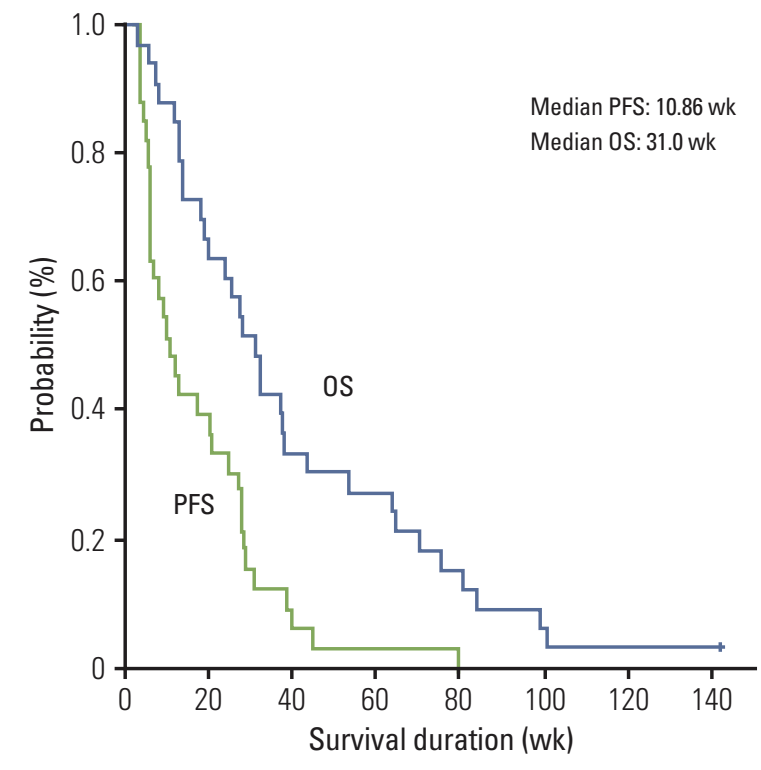

Fig. 1. Progression-free survival (PFS) and overall survival (OS).

response rates to second-line therapy are less than $10 \%$, and the median OS from the start of a second-line therapy is 4 months. Response rates to combination chemotherapy are higher than those to a single agent; however, the reported response rates are variable, reflecting the population heterogeneity [18].

Our study began in 2005, and a similar study's report was published in 2006. They showed that weekly paclitaxel and gemcitabine treatment were moderately active in SCLC patients pretreated with platinum and etoposide [19]. Paclitaxel $\left(80 \mathrm{mg} / \mathrm{m}^{2}\right)$ was given on days 1,8 , and 15 , and gemcitabine $\left(1,000 \mathrm{mg} / \mathrm{m}^{2}\right)$ was given on days 1 and 8 every 3 weeks. Of these patients, $32 \%$ had refractory disease, and the objective response rate was $26 \%$, including $20 \%$ in patients with refractory SCLC and $28.6 \%$ in patients with sensitive SCLC. The response rate was only slightly higher in patients with sensitive relapse than in those with refractory relapse, indicating that the regimen may be more useful in the former group. Predictive of response to second-line chemotherapy were the interval between the completion of induction and relapse, the extent of tumor regression achieved with the induction regimen, and the composition of the induction program. In the current study, the patient population was different from that previous study. Platinum plus irinotecan has been the standard first-line chemotherapy for SCLC at the National Cancer Center in Korea since 2002, when the Japanese Cooperative Oncology Group showed that irinote- can plus cisplatin was superior to etoposide plus cisplatin in terms of response rate, progression-free survival (PFS), and OS [20]. In this study, 30 patients $(91 \%)$ received irinotecan plus platinum and three patients received etoposide plus platinum. Sixteen patients $(49 \%)$ had refractory relapse. Given the refractory nature of the disease, the overall response rate of $30.3 \%$ in the current study is remarkable, although we set our initial target response rate to be $40 \%$. Our study design required at least 11 objective responses among 33 patients for this regimen to be regarded as worthy of further investigation; 10 patients achieved PR. We observed similar response rates in both groups $(29.4 \%$ in sensitive relapse and $31.3 \%$ in refractory relapse). These results suggest that this regimen may work in both groups. Also, high activity in patients with refractory disease may reflect a lack of adverse effects of prior treatment with irinotecan/platinum on the antitumor activity of paclitaxel/gemcitabine, which suggests a lack of cross-resistance between paclitaxel/gemcitabine and irinotecan/platinum. A recent randomized phase 3 trial of amrubicin versus topotecan as second-line treatment [21] reported a median OS of 7.5 months with amrubicin versus 7.8 months with topotecan; in refractory patients, median OS was 6.2 and 5.7 months, respectively. Median PFS was 4.1 months with amrubicin and 3.5 months with topotecan. Overall response rate was $31.1 \%$ with amrubicin and $16.9 \%$ with topotecan [21]. In this study, although amrubicin did not improve survival when compared with topotecan in the second-line treatment of patients with SCLC, OS did not differ significantly between treatment groups and improvement in OS was noted in patients with refractory disease treated with amrubicin. Although direct comparison of phase 2 data with phase 3 data is not reasonable, the weekly paclitaxel/gemcitabine regimen in the current study showed efficacy comparable to amrubicin and topotecan.

Because second-line chemotherapy in SCLC patients is only palliative, we have to consider quality of life, toxicity, and cost. In the second-line setting, all patients had already received platinum doublets, and thus, we had to consider cumulative toxic effects. A randomized phase III trial comparing oral topotecan $\left(2.3 \mathrm{mg} / \mathrm{m}^{2} /\right.$ day for 5 days every 21 days) to intravenous topotecan $\left(1.5 \mathrm{mg} / \mathrm{m}^{2} /\right.$ day for 5 days every 21 days), found reasonable response rates (18.3\% vs. $21.9 \%$ ) [22]. However, G4 neutropenia was common (47\% vs. $64.2 \%$ ), and diarrhea of all grades was observed (35.9\% vs. $19.9 \%$ ). A study of second-line weekly paclitaxel and gemcitabine showed G3-4 asthenia in 13\% of patients [19]. The average relative dose intensity was $72 \%$ for paclitaxel and $83 \%$ for gemcitabine. In the current study, the dose intensity was $86.4 \%$ for paclitaxel and $85.4 \%$ for gemcitabine, and treatment-related toxicity was observed considerably less often (18.2\% G4 neutropenia and 24.2\% G3 thrombocytope- 
Table 3. Tumor response by characteristics $(n=33)$

\begin{tabular}{|c|c|c|c|}
\hline Characteristic & PR $(n=10)$ & $S D+P D(n=23)$ & p-value \\
\hline \multicolumn{4}{|l|}{ Sex } \\
\hline Male & $9(90.0)$ & $21(91.3)$ & $>0.999$ \\
\hline Female & $1(10.0)$ & $2(8.7)$ & \\
\hline Age (yr) & $66(48-72)$ & $64(38-77)$ & \\
\hline \multicolumn{4}{|l|}{ ECOG PS } \\
\hline 0 & $3(30.0)$ & $3(13.0)$ & 0.137 \\
\hline $1-2$ & $7(70.0)$ & $20(87.0)$ & \\
\hline \multicolumn{4}{|c|}{ Stage (diagnosis) } \\
\hline Limited & $3(30.0)$ & $7(30.4)$ & $>0.999$ \\
\hline Extensive & $7(70.0)$ & $16(69.6)$ & \\
\hline \multicolumn{4}{|c|}{ Stage (enrollment) } \\
\hline Recurrence & $8(80.0)$ & 17 (73.9) & $>0.999$ \\
\hline Metastatic & $2(20.0)$ & $6(26.1)$ & \\
\hline \multicolumn{4}{|c|}{ Smoking history } \\
\hline Never & $1(10.0)$ & $2(8.7)$ & $>0.999$ \\
\hline Ever & $9(90.0)$ & $21(91.3)$ & \\
\hline \multicolumn{4}{|c|}{ Prior treated therapy } \\
\hline Chemo only & $6(60.0)$ & $16(69.6)$ & 0.845 \\
\hline CCRT & $3(30.0)$ & $5(21.7)$ & \\
\hline Chemo $\rightarrow$ RT & $1(10.0)$ & $2(8.7)$ & \\
\hline \multicolumn{4}{|c|}{ Best response of any prior treatments } \\
\hline $\mathrm{CR}+\mathrm{PR}$ & $10(100)$ & $19(82.6)$ & $>0.999$ \\
\hline $\mathrm{SD}+\mathrm{PD}$ & 0 & $4(17.4)$ & \\
\hline \multicolumn{4}{|c|}{ Chemotherapy-free interval (mo) ${ }^{\mathrm{a})}$} \\
\hline$<3$ & $4(40.0)$ & $13(56.5)$ & 0.465 \\
\hline$\geq 3$ & $6(60.0)$ & $10(43.5)$ & \\
\hline
\end{tabular}

Values are presented as number (\%) or median (range). PR, partial response; SD, stable disease; PD, progressive disease; ECOG PS, Eastern Cooperative Oncology Group performance status; CCRT, concurrent chemoradiotherapy; RT, radiotherapy; $\mathrm{CR}$, complete response. ${ }^{\mathrm{a})} \mathrm{Chemotherapy-free} \mathrm{interval:} \mathrm{from} \mathrm{the} \mathrm{last} \mathrm{date} \mathrm{of} \mathrm{prior} \mathrm{chemotherapy} \mathrm{to} \mathrm{start} \mathrm{date} \mathrm{of} \mathrm{pacli-}$ taxel-gemcitabine.

nia). Aside from myelosuppression, G3 non-hematologic toxicities were rare, and most of them were manageable with good compliance.

\section{Conclusion}

In conclusion, second-line paclitaxel and gemcitabine were well-tolerated with a $30.3 \%$ response rate in SCLC patients previously treated with platinum-based chemotherapy. Our study suggests that paclitaxel and gemcitabine can be good options for second-line therapy in platinum-resistant SCLC patients.

\section{Conflicts of Interest}

Paclitaxel (Padexol) and gemcitabine (Gero-am) were provided by Shinpoong Pharmaceutical Co., Seoul, Korea.

\section{Acknowledgments}

This work was supported by NCC Grants 0510140 and 0810090 from the National Cancer Center, Korea. 
Table 4. Hematologic and non-hematologic toxicity (NCI-CTC grade 3.0) (n=33)

\begin{tabular}{|c|c|c|c|c|c|c|}
\hline \multirow{2}{*}{ Toxicity } & \multicolumn{6}{|c|}{ NCI-CTC grade } \\
\hline & 0 & 1 & 2 & 3 & 4 & 5 \\
\hline \multicolumn{7}{|l|}{ Hematologic } \\
\hline Leukocytopenia & 0 & $8(25.8)$ & $14(42.4)$ & $7(21.2)$ & $2(6.1)$ & 0 \\
\hline Neutropenia & $3(9.1)$ & $3(9.1)$ & $7(21.2)$ & $14(42.4)$ & $6(18.2)$ & 0 \\
\hline Anemia & 0 & $6(18.2)$ & $21(63.6)$ & $6(18.2)$ & 0 & 0 \\
\hline Thrombocytopenia & $4(12.1)$ & $14(42.4)$ & 7 (21.2) & $8(24.2)$ & 0 & 0 \\
\hline \multicolumn{7}{|l|}{ Non-hematologic } \\
\hline \multicolumn{7}{|l|}{ Neurology } \\
\hline Neuropathy & $9(27.3)$ & $14(42.4)$ & $8(24.2)$ & $2(6.1)$ & 0 & 0 \\
\hline Insomnia & $31(93.9)$ & $2(6.1)$ & 0 & 0 & 0 & 0 \\
\hline \multicolumn{7}{|l|}{ GI } \\
\hline Stomatitis & $15(45.5)$ & $15(15.5)$ & $3(9.1)$ & 0 & 0 & 0 \\
\hline Anorexia & $8(24.2)$ & $21(63.63)$ & $4(12.1)$ & 0 & 0 & 0 \\
\hline Nausea/vomiting & $21(63.6)$ & $12(36.4)$ & 0 & 0 & 0 & 0 \\
\hline Constipation & $26(78.8)$ & $5(15.2)$ & $2(6.1)$ & 0 & 0 & 0 \\
\hline Diarrhea & $24(72.7)$ & $7(21.2)$ & $2(6.1)$ & 0 & 0 & 0 \\
\hline \multicolumn{7}{|l|}{ Hepatic } \\
\hline AST & $20(60.6)$ & $9(27.3)$ & $3(9.1)$ & $1(3.0)$ & 0 & 0 \\
\hline ALT & $13(87.9)$ & $18(54.5)$ & $2(6.1)$ & 0 & 0 & 0 \\
\hline Bilirubin & $29(87.9)$ & $3(9.1)$ & $1(3.0)$ & 0 & 0 & 0 \\
\hline \multicolumn{7}{|l|}{ Renal } \\
\hline Creatinine & $23(69.7)$ & $8(24.22)$ & $2(6.1)$ & 0 & 0 & 0 \\
\hline \multicolumn{7}{|l|}{ Dermatology } \\
\hline Alopecia & $4(12.1)$ & $12(36.4)$ & $17(51.5)$ & 0 & 0 & 0 \\
\hline Rash & $30(90.9)$ & $3(9.1)$ & 0 & 0 & 0 & 0 \\
\hline Itching & $27(81.8)$ & $3(9.1)$ & $3(9.1)$ & 0 & 0 & 0 \\
\hline \multicolumn{7}{|l|}{ Others } \\
\hline Asthenia & $9(27.3)$ & $15(45.5)$ & $7(21.2)$ & $1(3.0)$ & 0 & 0 \\
\hline Myalgia & $11(33.3)$ & $12(36.4)$ & $10(30.3)$ & 0 & 0 & 0 \\
\hline Edema & $31(93.9)$ & $2(6.1)$ & 0 & 0 & 0 & 0 \\
\hline Infection & $31(93.9)$ & 0 & $1(3.0)$ & 0 & 0 & $1(3.0)$ \\
\hline Febrile neutropenia & $32(97.0)$ & 0 & 0 & $1(3.0)$ & 0 & 0 \\
\hline Fever & $26(78.8)$ & $5(15.2)$ & $2(6.1)$ & 0 & 0 & 0 \\
\hline Pneumonitis & $31(93.9)$ & 0 & 0 & $2(6.1)$ & 0 & 0 \\
\hline
\end{tabular}

Values are presented as number (\%). NCI-CTC, National Cancer Institute Common Toxicity Criteria; GI, gastrointestinal; AST, aspartate aminotransferase; ALT, alanine aminotransferase.

\section{References}

1. Perez-Soler R, Glisson BS, Lee JS, Fossella FV, Murphy WK, Shin DM, et al. Treatment of patients with small-cell lung cancer refractory to etoposide and cisplatin with the topoisomerase I poison topotecan. J Clin Oncol. 1996;14:2785-90.

2. Furuse K, Kubota K, Kawahara M, Takada M, Kimura I, Fujii $\mathrm{M}$, et al. Phase II study of vinorelbine in heavily previously treated small cell lung cancer. Japan Lung Cancer Vinorelbine Study Group. Oncology. 1996;53:169-72.

3. Smit EF, Fokkema E, Biesma B, Groen HJ, Snoek W, Postmus
PE. A phase II study of paclitaxel in heavily pretreated patients with small-cell lung cancer. Br J Cancer. 1998;77:347-51.

4. van der Lee I, Smit EF, van Putten JW, Groen HJ, Schlosser NJ, Postmus PE, et al. Single-agent gemcitabine in patients with resistant small-cell lung cancer. Ann Oncol. 2001;12:557-61.

5 . Eckardt JR. Topotecan in relapsed small-cell lung cancer: can good things come in small packages? Clin Lung Cancer. 2003;4:229-30.

6. Ardizzoni A, Hansen H, Dombernowsky P, Gamucci T, 
Kaplan S, Postmus P, et al. Topotecan, a new active drug in the second-line treatment of small-cell lung cancer: a phase II study in patients with refractory and sensitive disease. The European Organization for Research and Treatment of Cancer Early Clinical Studies Group and New Drug Development Office, and the Lung Cancer Cooperative Group. J Clin Oncol. 1997;15:2090-6.

7. Ardizzoni A, Manegold C, Debruyne C, Gaafar R, Buchholz E, Smit EF, et al. European Organization for Research and Treatment of Cancer (EORTC) 08957 phase II study of topotecan in combination with cisplatin as second-line treatment of refractory and sensitive small cell lung cancer. Clin Cancer Res. 2003;9:143-50.

8. Yamamoto N, Tsurutani J, Yoshimura N, Asai G, Moriyama A, Nakagawa K, et al. Phase II study of weekly paclitaxel for relapsed and refractory small cell lung cancer. Anticancer Res. 2006;26:777-81.

9. Masters GA, Declerck L, Blanke C, Sandler A, DeVore R, Miller $\mathrm{K}$, et al. Phase II trial of gemcitabine in refractory or relapsed small-cell lung cancer: Eastern Cooperative Oncology Group Trial 1597. J Clin Oncol. 2003;21:1550-5.

10. Edelman MJ, Quam H, Mullins B. Interactions of gemcitabine, carboplatin and paclitaxel in molecularly defined non-smallcell lung cancer cell lines. Cancer Chemother Pharmacol. 2001;48:141-4

11. Shord SS, Faucette SR, Gillenwater HH, Pescatore SL, Hawke RL, Socinski MA, et al. Gemcitabine pharmacokinetics and interaction with paclitaxel in patients with advanced nonsmall-cell lung cancer. Cancer Chemother Pharmacol. 2003;51: 328-36.

12. Hirsh V, Whittom R, Ofiara L, Desjardins P, Ayoub J, Charpentier $\mathrm{D}$, et al. Weekly paclitaxel and gemcitabine chemotherapy for metastatic non-small cell lung carcinoma (NSCLC): a dose-optimizing phase II trial. Cancer. 2003;97:2242-7.

13. Recchia F, Saggio G, Cesta A, Candeloro G, Rea S. Paclitaxel plus gemcitabine in advanced non-small cell lung cancer patients with low performance status. Anticancer Res. 2005;25: 409-14.
14. Gillenwater HH, Stinchcombe TE, Qaqish BF, Tyann M, Hensing TA, Socinski MA. A phase II trial of weekly paclitaxel and gemctiabine infused at a constant rate in patients with advanced non-small cell lung cancer. Lung Cancer. 2005;47: 413-9.

15. Therasse P, Arbuck SG, Eisenhauer EA, Wanders J, Kaplan RS, Rubinstein L, et al. New guidelines to evaluate the response to treatment in solid tumors. European Organization for Research and Treatment of Cancer, National Cancer Institute of the United States, National Cancer Institute of Canada. J Natl Cancer Inst. 2000;92:205-16.

16. Simon R. Optimal two-stage designs for phase II clinical trials. Control Clin Trials. 1989;10:1-10.

17. O'Brien ME, Ciuleanu TE, Tsekov H, Shparyk Y, Cucevia B, Juhasz $\mathrm{G}$, et al. Phase III trial comparing supportive care alone with supportive care with oral topotecan in patients with relapsed small-cell lung cancer. J Clin Oncol. 2006;24:5441-7.

18. Nair BS, Bhanderi V, Jafri SH. Current and emerging pharmacotherapies for the treatment of relapsed small cell lung cancer. Clin Med Insights Oncol. 2011;5:223-34.

19. Dongiovanni V, Buffoni L, Berruti A, Dongiovanni D, Grillo $\mathrm{R}$, Barone $\mathrm{C}$, et al. Second-line chemotherapy with weekly paclitaxel and gemcitabine in patients with small-cell lung cancer pretreated with platinum and etoposide: a single institution phase II trial. Cancer Chemother Pharmacol. 2006;58: 203-9.

20. Noda K, Nishiwaki Y, Kawahara M, Negoro S, Sugiura T, Yokoyama A, et al. Irinotecan plus cisplatin compared with etoposide plus cisplatin for extensive small-cell lung cancer. N Engl J Med. 2002;346:85-91.

21. von Pawel J, Jotte R, Spigel DR, O'Brien ME, Socinski MA, Mezger J, et al. Randomized phase III trial of amrubicin versus topotecan as second-line treatment for patients with small-cell lung cancer. J Clin Oncol. 2014;32:4012-9.

22. Eckardt JR, von Pawel J, Pujol JL, Papai Z, Quoix E, Ardizzoni A, et al. Phase III study of oral compared with intravenous topotecan as second-line therapy in small-cell lung cancer. J Clin Oncol. 2007;25:2086-92. 